\title{
Spontaneous Rupture of Uterine Veins In Pregnancy
}

\author{
KH.SHAHNEWAJ ${ }^{1}$ MD. AL SAYED ${ }^{2}$ KAUSER JABIN ${ }^{3}$
}

\begin{abstract}
Summary:
Spontaneous rupture of uterine vessels during pregnancy is a rare cause of shock that has a high rate of maternal and foetal loss. Though the usual presentation is of maternal shock, it is necessary to have in mind that possibility of blood vessels rupture may be in cases of abdominal pain \& hypotension of unknown origin during pregnancy.

In such an event an exploratory laparatomy followed by caesarean with ligation of the uterine vessel or its branch can help in preventing a maternal mortality. The advent of modern resuscitative facilities, anaesthesia techniques have lowered mortality associated with this condition from $49 \%$ to $3.6 \%$.
\end{abstract}

\section{Introduction:}

Hemoperitoneum during pregnancy resulting from spontaneous rupture of uterine varicose veins is potentially lethal complication, associated with high fetal and maternal mortality ${ }^{1-11}$. This is likely to be misdiagnosed because of its rarity. Presenting symptoms include severe abdominal pain, followed rapidly by maternal shock and fetal distress. Approximately 120 cases have been reported in literature, first case was reported in 1778. Majority of ruptures $(61 \%)$ were diagnosed before the onset of labour, and $21 \%$ occurred in post partum period. We report a case of $26 \mathrm{yrs}$ old woman at $30 \mathrm{wks}$ of gestation, who had acute abdomen and shock because of spontaneous rupture of uterine varicose veins.

\section{Case Report:}

A $26 \mathrm{yr}$ old woman, primigravidas first presented at 30 wks gestation with history of chicken pox and threatened preterm labour. Tocolysis was done; she received 2 doses of injection dexamethasone, but left against medical advice 12-hours after admission. After one week presented with same complaints, but left just after 10 mins without treatment.

On her last admission she presented with vomiting, pain lower abdomen and dizziness. Her B.P was 110/ $70 \mathrm{~mm} / \mathrm{Hg}$; pulse was 90/min and was afebrile. Fetal heart rate was $160 / \mathrm{min}$. Marked pallor was noted this time. Ultarsound done but no evidence of abruptio placentae found, fetal movements were seen. Blood samples were taken for cross matching and baseline workup. Suddenly patient had a syncopal attack and
B.P dropped to $60 / 40 \mathrm{~mm} / \mathrm{Hg}$. She was resuscitated with I/V fluids and received blood transfusion. Ultrasonography repeated by consultant radiologist later on, this time fetal cardiac activity was absent and again no sign of placental abruption was evident. Patient's condition deteriorated over time, with markedly increasing pallor, tachycardia and hypotension. She was shifted to ICU, with a provisional diagnosis of septicemia (keeping in view her history of chicken pox), and placental abruption was still not ruled out categorically and received packed cell transfusion, broad-spectrum antibiotics. In ICU patient complained of severe epigastric pain. USG hepatobillary system was requested and this time radiologist noticed significant amount of free fluid in peritoneal cavity, Ultrasound guided aspiration was done, and frank blood was aspirated. Surgical team was consulted and exploratory Laparatomy planned with provisional diagnosis of ruptured splenic artery aneurysm. Abdomen was entered through midline incision, and about $2000 \mathrm{ml}$ blood removed from peritoneal cavity. Cesarean section done and dead female fetus delivered. No obvious source of bleeding encountered, all abdominal and pelvic viscera were intact. The only abnormal finding was dilated tortuous varicose veins on uterine fundus with active ooze from few veins, these vessels were ligated. Patient was stabilized with 4units packed cell and 4- FFPs transfusion.

Patient had uneventful recovery and was discharged in stable condition on $10^{\text {th }}$ post operative day.

In the following year she had uneventful pregnancy delivered at term by caesarean section, healthy alive baby. 


\section{Discussion:}

This condition has a relatively infrequent incidence and has been associated in the past with very high maternal and fetal mortality. The obstetrician is faced with both a puzzling diagnostic dilemma and a perplexing management scheme.

This potentially lethal complication of pregnancy is so rare that it is not described in standard textbooks. Few obstetricians see even a single case.

Placental abruption is the frequent misdiagnosis ${ }^{2}$. Ignorance of this condition may lead to delay in diagnosis with possible tragic consequences.

The etiology is unknown, but Hodgkins and Christiansen suggested that rupture results from a sudden rise in venous pressure in varicose hypertrophied, utero-ovarian vessels. These veins are predisposed to rupture because of their tortuous path, lack of valves, and distension during pregnancy, because of increase in blood volume and cardiac output.

Majority of ruptures occur in labour, as was seen in this case, as she presented with preterm labour pains. Sudden increase in the venous pressure as in labour, coitus, defecation and weight lifting may play a role in spontaneous rupture. Previous surgeries on uterus, leiomyoma and multiple pregnancies have been associated with this condition.

Hemoperitoneum or broad ligament and retroperitoneal hematoma follow the rupture. In either event acute pain abdomen is the first symptom, quickly followed by shock. Ultrasound scan is useful to exclude placental abruption, detect free fluid and guide paracentesis needle.

Spontaneous Hemoperitoneum in pregnancy has also been reported from rupture of various abdominal and pelvic organs and aneurysmal vessels.

Treatment consists of aggressive fluid and blood replacement, together with prompt surgical intervention, provide the only chance for a favorable outcome for both fetus and mother. Cesarean section is required in late pregnancy for adequate exploration. In early pregnancy ligation of vessels is recommended. Even with these interventions fetal mortality remains high as $31 \%$, though with the advent of modern resuscitative facilities and anesthesia techniques maternal mortality has been reduced to $3.6 \%{ }^{2}$.

\section{Conclusion:}

When a pregnant woman experiences acute onset, severe abdominal pain even without any history of abdominal trauma ruptured uterine varicose veins and hemoperitoneum should be included in the differential diagnosis. Recurrence in a subsequent pregnancy is unknown.

\section{References:}

1. Choobun, Pinjaroen MD,Chatpavit Getpook MD.Spontaneous Intra-Abdominal bleeding during pregnancy Med Assoc Thai 2006;87(7):1044-6

2. Ginsburg KA, Valdes C,Schnider G. Spontaneous utero-ovarian vessels rupture during pregnancy:three case reports and a review of the literature.Obstet Gynecol 1987;69:474-6.

3. Foley MR,Sonek JD and Lavender M,Zuspal FP.Spontaneous rupture of utero-ovarian veins in pregnancy:two case reports.Am J of Obstet Gynecol 1987;156:962-4.

4. Hodgkinson CP,Christensen RC. Haemorrhage from ruptured utero-ovarian veins during pregnancy.Am $\mathrm{J}$ of Obstet Gynecol 1950;59:1112-1117

5. Simpson I,Ng A, Griffin C.A rare cause of an acute abdomen in late pregnancy.Aust NZ J Obstet Gynecol 1995;35:435-6.

6. Fiori O,Pruqnolles H,Darai E. Spontenous uterine artery rupture during pregnancy in a woman with sickle cell disease:a case report.J Reprod Med.2007;52(7):657-8.

7. Aziz U,Kulkarni A, Lazic D,Cullimore G.Spontaneous rupture of the uterine vessels in pregnancy.Obstet Gynecol 2004;103:1089-91.

8. Swaegers MC,Hauspy JJ,Buytaert PM,DeMaeseneer MG.Spontaneous rupture of the uterine artery in pregnancy.Eur.J Obstet Gynecol Reprod Biol 1997;75:145-6.

9. Arie Koifman, Adi Y.Weintraub,David Segal.Idiopathic spontaneous hemoperitoneum during pregnancy.Archives of Gynecology and Obstetrics 2006;276:269-70.

10. Wu CY,Hwanq JL,Liu YH,Hsieu BC.Spontaneous hemoperitoneum in pregnancy from a ruptured superficial uterine vessel.Taiwan $\mathrm{J}$ Obstet Gynecol 2007;46:77-80.

11. Hashimoto K,Tabata C,Ueno Y,Fukuda G.Sponteneous rupture of uterine surface varicose veins in pregnancy: a case report.J Reprod Med 2006; 51:722-4. 